was replaced with aspirin. Echocardiogram revealed a small patent foramen ovale. Lipoprotein (a) level was elevated, and he was heterozygous for MTHFR C677T mutation. Recovery was almost complete after one month. Case 3. A 7-year-old boy had acute right-sided numbness and tingling $3 \mathrm{~h}$ after a karate chop to his neck. In the ER 1 hour later he had flaccid right-sided weakness of face, arm and leg. MRI showed infarcts in left parietal lobe, left medial medulla, and right cerebellum. DSA showed right vertebral artery dissection and occlusion. He recovered slowly and had no weakness at 1 year. (Sepelyak K, Gailloud P, Jordan LC. Athletics, minor trauma, and pediatric arterial ischemic stroke. Eur J Pediatr May 2010;169:557-562).(LC Jordan MD, Dept Pediatrics, Johns Hopkins University School of Medicine, $200 \mathrm{~N}$ Wolfe St, Suite 2158, Baltimore, MD 21287. E-mail: Ljordan2@jhmi.edu).

COMMENT. Diagnosis of arterial ischemic stroke following minor athletic trauma is often delayed, with risk of progression or recurrence. Arterial dissection is associated in up to $20 \%$ cases. Treatment with anticoagulation is usually continued for 3 to 6 months (Roach ES et al. Stroke 2008;39:2644-2691). If dissection is not visualized by DSA, the Johns Hopkins authors recommend discontinuing anticoagulants and replacing with aspirin at time of discharge. Some restrictions on contact sports participation are generally followed. Recurrence risks of up to $30 \%$ are reported.

Endovascular therapy in pediatric intracranial carotid artery dissection is reported in a 12-year-old boy. (Lai Y-J et al. Pediatr Neurol April 2010;42(4):291-294). Intraarterial thrombolysis and stent reconstruction successfully recanalized the occluded arterial segment.

\title{
GRADENIGO'S SYNDROME WITH LATERAL VENOUS SINUS THROMBOSIS
}

A 4-year-old girl was admitted to the Department of Pediatrics, University of Chieti, Italy, with a 2-week history of fever and right otitis media, headache and vomiting. Exam showed right facial and abducens nerve palsies. CT scan showed a right mastoiditis. MRI revealed ipsilateral sinus thrombophlebitis. Complete resolution followed treatment with high dose IV antibintics and oral anticoagulants. (Scardapane A, Del Torto M, Nozzi M, Elio C, Breda L, Chiarelli F. Gradenigo's syndrome with lateral venous sinus thrombosis: successful conservative treatment. Eur J Pediatr April 2010;169:437-440). (Dr A Scardapane.E-mail: a.scardapane@gmail.com).

COMMENT. Otitis media, pain in the distribution of the first and second branches of the trigeminal nerve, and ipsilateral abducens palsy are the three characteristic signs of Gradenigo's syndrome. Facial nerve palsy occurs in severe cases, as above. Otitis media and inflammation spread to involve the petrous apex, dura and lateral venous sinus. Irritation of the ophthalmic branch of the trigeminal nerve and sixth nerve follow. The nerve involvement may be delayed for 1 to 3 months. The syndrome is now rare, but early diagnosis is important, to avoid necessity for surgery. 